\title{
2341. Acoustic calculation in low frequency sonopheresis based on bubble dynamics
}

\author{
Hanmin Peng ${ }^{1}$, Penghui $\mathrm{Lu}^{2}$, Pancheng $\mathrm{Zhu}^{3}$, Boping $\mathrm{Yu}^{4}$ \\ State Key Lab of Mechanics and Control of Mechanical Structures, \\ Nanjing University of Aeronautics and Astronautics, Nanjing, 210016, China \\ ${ }^{1}$ Corresponding author

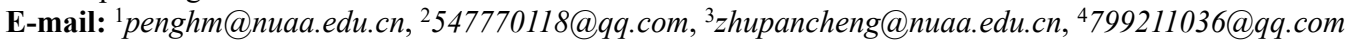

Received 8 April 2016; received in revised form 1 September 2016; accepted 9 September 2016 DOI https://doi.org/10.21595/jve.2016.17054

Check for updates

\begin{abstract}
As a type of transdermal permeability enhancement, low frequency sonophoresis (LFS) has been studied for more than twenty years. The acoustic pressure in LFS is a crucial ultrasonic parameter to improve the permeability, but it is difficult to measure in the drug donor because of its small size and narrow shape. In this paper, an acoustic-piezoelectric coupling model is established based on bubble dynamics, which can be utilized to calculate the acoustic pressure distributions in LFS using a commercial finite element software called COMSOL multiphysics. The calculated results of acoustic pressure are in accordance with the measured values, so this model has great potential for theoretical analyses in acoustic fields of LFS. Calculated and experimental results show that the maximum acoustic pressure is under the transducer's head, and the value dropped as away from the head due to the acoustic attenuation caused by cavitation; the transducer head should be closer to the skin to obtain larger acoustic pressure on the skin. Therefore, this model can be used to simulate and analyze the characteristics of acoustic fields, as a theoretical tool for the structural design of the ultrasonic transducer applied in LFS.
\end{abstract}

Keywords: acoustic, sonopheresis, low frequency ultrasound, numerical simulation, bubble dynamics.

\section{Nomenclature}

$b \quad$ Damping factor in gas-liquid medium

c $\quad$ Speed of sound $\left(1450 \mathrm{~m} \cdot \mathrm{s}^{-1}\right.$ in the liquid)

$c_{c} \quad$ Complex acoustic speed

$C_{\text {coe }} \quad$ Parameter chosen to match the gas volume void fraction $\beta$

$d \quad$ Skin thickness $\left(5.62 \times 10^{-4} \mathrm{~m}\right)$

$d \quad$ Piezoelectric constants

D Electric displacement

E $\quad$ Electric field

$f \quad$ The working frequency of ultrasonic transducer [21000 Hz]

$F_{A} \quad$ Load (force per unit area) experienced by the structure

$k_{e q} \quad$ Equivalent wavenumber in the gas-liquid medium

$k_{\alpha} \quad$ Equivalent wavenumber in the acoustic field

$n \quad$ The surface normal

$P \quad$ Acoustic pressure

$p_{0} \quad$ Initial amplitude of acoustic pressure

$p_{t} \quad$ Total acoustic pressure

$p_{b} \quad$ Undisturbed pressure in the liquid

$p_{\infty} \quad$ Equilibrium pressure in the liquid

$Q_{d} \quad$ Dielectric loss of the piezoelectric ceramics

$Q_{m} \quad$ Mechanical loss of the ultrasonic transducer

$r \quad$ Radial coordinate $r$

$R \quad$ Equilibrium radium of cavitation bubbles

$R_{0} \quad$ Radius for the Gaussian radii distribution with a maximum value $\left(5 \times 10^{-4} \mathrm{~m}\right)$

$R_{1} \quad$ Minimum bubble radius $\left(5 \times 10^{-6} \mathrm{~m}\right)$

$R_{2} \quad$ Maximum bubble radius $\left(3 \times 10^{-3} \mathrm{~m}\right)$ 


$\begin{array}{ll}R_{\text {instant }} & \text { Instantaneous bubble radius } \\ S^{E} & \text { Flexibility coefficient } \\ S & \text { Strain component } \\ t & \text { Time } \\ T & \text { Stress component } \\ u & \text { Velocity field } \\ u_{t t} & \text { Structural acceleration } \\ V_{0 p} & \text { Zero-to-peak voltage } \\ x & X \text { direction of Cartier coordinate system shown in Figs. } 5 \text { and } 7(\mathrm{a}) \\ y & Y \text { direction of Cartier coordinate system shown in Figs. } 5 \text { and } 7(\mathrm{a}) \\ z & \text { Z direction of Cartier coordinate system shown in Figs. } 5 \text { and } 7(\mathrm{a}) \\ \alpha & \text { Attenuation coefficient } \\ \alpha_{2} & \text { Absorption coefficient } \\ \beta & \text { Gas volume void fraction } \\ \gamma & \text { Specific heat ratio of the gas inside the bubbles }(1.4) \\ \varepsilon^{T} & \text { Free dielectric constant } \\ \varepsilon_{p} & \text { Relative dielectric constant } \\ \eta_{s} & \text { Mechanical loss factor } \\ \eta_{e} & \text { Dielectric loss factor } \\ \mu_{l} & \text { The viscosity of the liquid }\left(6.51 \times 10^{-2} \mathrm{~Pa} \cdot \mathrm{s} \text { with } 40^{\circ} \mathrm{C} \text { water }\right) \\ \rho & \text { Material density } \\ \rho_{c} & \text { Complex density } \\ \sigma_{l} & \text { Surface tension of the liquid }\left(69.56 \times 10^{-3} \mathrm{~N} / \mathrm{m} \text { with } 40{ }^{\circ} \mathrm{C}\right) \\ \sigma_{S D} & \text { Standard deviation of bubbles distribution }\left(2 \times 10^{-3} \mathrm{~m}\right) \\ \Phi & \text { Complex dimensionless parameters } \\ \chi & \text { A coefficient in Eq. }(12) \\ \omega & \text { Angular frequency of the ultrasonic transducer } \\ \omega_{0} & \text { Resonance frequency of bubbles } \\ \omega_{b} & \text { Blood perfusion rate } \\ & \end{array}$

\section{Introduction}

With rapid development of therapeutic ultrasound, a type of transdermal drug delivery system called low frequency sonophoresis (LFS) has attracted more attention in the last two decades $[1,2]$. LFS $(20-100 \mathrm{kHz})$ can effectively improve the transdermal permeability of different drugs, not only including hydrophobic permeants but also hydrophilic ones [3, 4]. In 1995, Samir Mitragotri, Blankschrein D. and Langer R. [5] completed a series of experiments to suggest that LFS can deliver and control three kinds of therapeutic protein namely insulin, interferon $\gamma$, erythropoietin through the skin. Untill 2010, Baris E. Polat, Blankschtein D., and Langer R. [4] have reviewed plenty of different drugs whose permeation through the stratum corneum has been enhanced. Recently, Mai Aldwaikat and Mohammed Alarjah [6] also found that ultrasound technology enhanced the permeation of diclofenac sodium through EpiDerm ${ }^{\mathrm{TM}}$ (a three-dimension viable skin) fivefold. As a whole, LFS is a valuable transdermal enhancing method, especially for hydrophobic permeants.

Although LFS has been proven effectively for improvement of transdermal drug delivery, its principle is still not fully understood [2]. Generally, if acoustic intensity is larger than cavitation threshold value (e.g. $1.3 \mathrm{~W} / \mathrm{cm}^{2}$ ), there would be plenty of cavitation bubbles in drug liquid of which the inertial cavitation is responsible for its transdermal permeability enhancement [7]; on the contrary, acoustic pressure plays a greater role on permeation improvement. However, it is difficult to obtain the acoustic distribution in LFS by experimental measurement, because traditional equipment such as measurement hydrophone is too large to be placed into the drug donor. Consequently, this paper will present an acoustic calculation method based on bubble dynamics to simulate the acoustic pressure and its distribution in an in-vitro LFS, and then two small sheets of polyvinylidene fluoride (PVDF) piezoelectric film are used to measure the 
appropriate pressure in the donor and receptor.

An acoustic-piezoelectric coupling model is built up using a commercial finite element (FEM) software call COMSOL Multiphysics, with which the absorption coefficients of liquid are calculated based on bubble dynamics in terms of Cafisch equations theory proposed by Kerry W. Commander [8]. Calculated upper-skin acoustic pressure is appropriately close to the experimental values, demonstrating the efficiency of this model. According the results, the acoustic pressure is maximal under the transducer's head, and quickly decays far away from the head; in order to obtain a larger acoustic pressure, the transducer should be put closer to the skin.

\section{Experimental setup}

An ultrasonic transducer was bought from Hainertec (Suzhou) Co., Ltd. (Suzhou, China). Fig. 1 shows the transducer structure used in the simulated and experimental analysis. Its transduction section has the same type and size as the Langevin. Four pieces of piezoelectric ceramic rings (PZT-8) packed an opposite polarization direction to each other were sandwiched by the back and front masses (titanium alloy) to form the transduction area. An aluminum alloy frame was assembled to hold the entire transduction section. The tip of the front mass, used as the acoustic exposure section, is named "transducer head" in this paper. When voltage was applied to the piezoelectric ceramics and the frame was fixed, the transducer head emitted ultrasonic waves into the liquid media. The vibration displacement on the top surface of the transducer head was measured by a PSV-500 scanning vibrometer from Polytec Co., Ltd. (Waldbronn, German). In this paper, the first mode of longitudinal vibration of the ultrasonic transducer is excited with $5.5 \mathrm{~W}$ input electric power at $21 \mathrm{kHz}$, whose value can produce acoustic cavitations in the liquid. More characteristics of this ultrasonic transducer can be seen in Fig. 5 of Section 4.

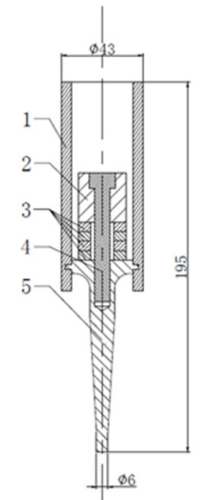

a) Structural sketch

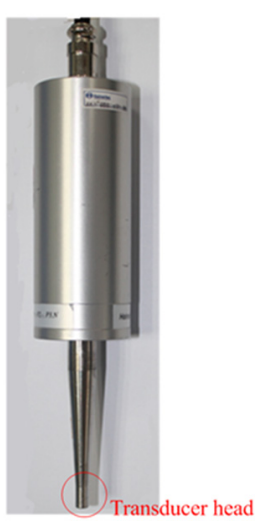

b) Setup

Fig. 1. Ultrasonic transducer: 1 - frame; 2 - back mass; 3 - piezoelectric ceramics; 4 - bolt; 5 - front mass (its top is transducer head)

The measurement system in in vitro LFS, shown in Fig. 2, is usually used to measure the permeation of Calcein against time, but in this paper, the acoustic pressure is also measured during the permeation within the same system. A rat skin was sectioned and mounted in vertical Franz diffusion cells TT-8(D) (15-mm inner diameter), which were obtained from Tianjin Rightway Technology Co., Ltd. (Tianjin, China). A Franz cell typically composes of a donor, skin sample, and receptor, as shown in Area A of Fig. 2. A constant temperature system kept the circulating water at $37^{\circ} \mathrm{C}$, and the drug liquid (Calcein with sodium lauryl sulfate solution in this paper) height in the donor maintained $0.025 \mathrm{~m}$.

As shown in Fig. 2, the traditional hydrophone and other acoustic sensors are too big to lay in the donor in in vitro LFS. Thus, in order to measure the acoustic pressure in a small space, two soft and small PVDF piezoelectric strips (from Jinzhou Kexin Electronic Materials Co., Ltd., 
China) were used. These films of PVDF piezoelectric material can sense external pressure change within the ultrasonic frequency band, and responses in a voltage that is proportional to the change.
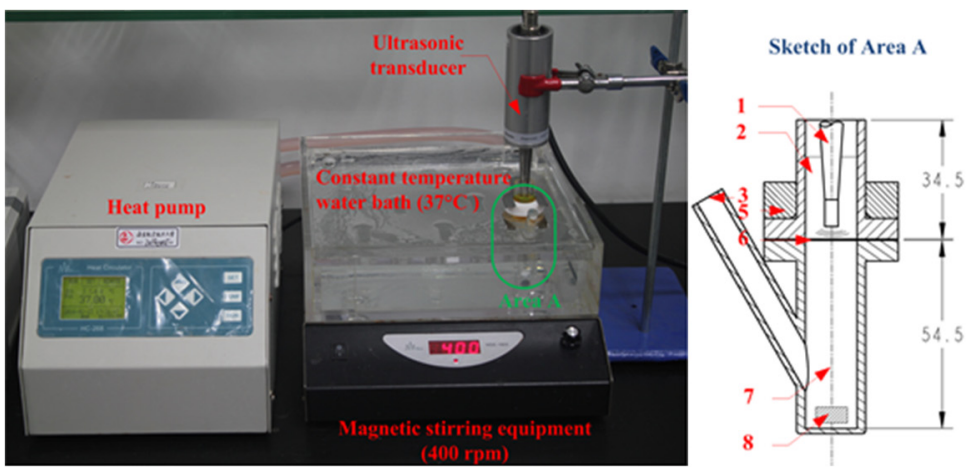

1.Transducer head; 2. Donor; 3. Liquid draw-off pipe; 4 . Thermocouple wire; 5. Teflon; 6. Skin; 7.Receptor; 8. Magnetic stirring bar

Fig. 2. Measurement system of permeation in in vitro LFS

In our experiments, the two strips were placed under the ultrasonic transducer in the donor and at the liquid draw-off position in the receptor respectively, as shown in Fig. 3(a). The former was used to measure the approximate acoustic pressure up the epidermis, and the latter was used to measure the pressure in the receptor. The relationship between the acoustic pressure $(P)$ and response voltage $\left(V_{0 p}\right)$ of the PVDF strips was calibrated by an acoustic intensity device obtained from Hangzhou Create Ultrasonic Technology Co., Ltd. (Hangzhou, China). In the calibration experiments, a 2 L tank full of water was used, in which the acoustic intensity device and PVDF piezoelectric strips were immersed at the same position and same time. So the acoustic pressure values measured with the acoustic intensity device were the same as that of the PVDF piezoelectric strips, which were proportional to their response voltage values. So, their linear relationship can be seen in Fig. 3(b), which are approximately expressed as:

$P=21763 V_{0 p}$

In the experiments, the response voltages of the two PVDF strips were collected by an oscilloscope (from Tektronix Inc., America), and then acoustic pressure was calculated according to Eq. (1). The PVDF strip in the donor was located between the skin and ultrasonic transducer, which was about $2 \mathrm{~mm}$ height upon the skin. Acoustic pressure at the same position was measured every $10 \mathrm{~min}$ during the first $90 \mathrm{~min}$ of LFS, and the average of these values (including $0 \mathrm{~Pa}$ at time $=0$ ) represented the value $P$.

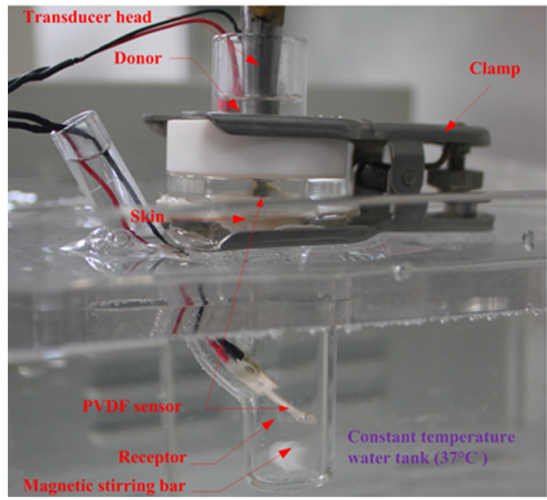

a)

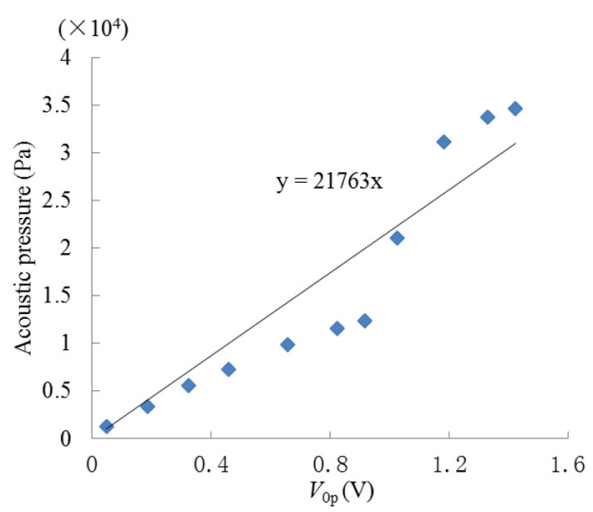

b)

Fig. 3. Measurement and calculated methods of acoustic pressure: a) measurement system; b) relationship between the response voltage (0-peak value) of PVDF strip and acoustic pressure 


\section{Experimental setup}

In LFS, an acoustic field with certain intensity in the donor produces acoustic bubbles. The bubbles can be classified into two categories, stable and inertial cavitation: for stable cavitation, the acoustic pressure and streams may increase the permeability of a drug outside the skin; meanwhile, the inertial cavitation gives rise to plenty of shock waves and the micro-jets physically penetrate the stratum corneum (SC), contributing to drug permeation through the skin. Furthermore, some parts of the skin are compressed and the others are stretched due to acoustic streams. In this paper, a FEM simulation method based on COMSOL is used to calculate the acoustic field distribution in in vitro LFS. Here, the simulation is conducted with the following main assumptions:

1) The acoustic field is assumed to be an attenuation of linear elastic waves in the lossy media, including the skin and liquids in the donor and receptor;

2) The influence of the liquid draw-off pipe on the acoustic field is ignored.

\subsection{Piezoelectric coupling}

For the Langevin type ultrasonic transducer shown in Fig. 1, the driving voltage is applied on four pieces of piezoelectric ceramics, and the converse piezoelectric effect can be calculated by classical piezoelectric equations [9]. When considering the mechanical loss and dielectric loss factors, it can be represented as (in linear elastic range):

$\left\{\begin{array}{l}\mathbf{S}=\mathbf{S}^{E} \mathbf{T}+\mathbf{d E}, \\ \mathbf{D}=\mathbf{d} \mathbf{T}+\boldsymbol{\varepsilon}^{T} \mathbf{E}\end{array}\right.$

where:

$\mathbf{S}=\left[\begin{array}{llllll}S_{1} & S_{2} & S_{3} & S_{4} & S_{5} & S_{6}\end{array}\right]^{T}, \quad \mathbf{D}=\left[\begin{array}{llll}D_{1} & D_{2} & D_{3}\end{array}\right]^{T}$,

$\mathbf{T}=\left[\begin{array}{llllll}T_{1} & T_{2} & T_{3} & T_{4} & T_{5} & T_{6}\end{array}\right]^{T}, \quad \mathbf{E}=\left[\begin{array}{llll}E_{1} & E_{2} & E_{3}\end{array}\right]^{T}$,

$\mathbf{s}^{E}=\left[\begin{array}{cccccc}s_{11} & s_{12} & s_{13} & 0 & 0 & 0 \\ s_{12} & s_{11} & s_{12} & 0 & 0 & 0 \\ s_{13} & s_{13} & s_{33} & 0 & 0 & 0 \\ 0 & 0 & 0 & s_{44} & 0 & 0 \\ 0 & 0 & 0 & 0 & s_{44} & 0 \\ 0 & 0 & 0 & 0 & 0 & 2\left(s_{11}-s_{12}\right)\end{array}\right], \quad \mathbf{d}=\left[\begin{array}{cccccc}0 & 0 & 0 & 0 & d_{15} & 0 \\ 0 & 0 & 0 & d_{15} & 0 & 0 \\ d_{31} & d_{31} & d_{33} & 0 & 0 & 0\end{array}\right]$,

$\boldsymbol{\varepsilon}^{T}=\left[\begin{array}{ccc}\varepsilon_{11} & 0 & 0 \\ 0 & \varepsilon_{11} & 0 \\ 0 & 0 & \varepsilon_{33}\end{array}\right]$,

where $\mathbf{S}$ is the strain component, $\mathbf{D}$ is the electric displacement, $\mathbf{S}^{E}$ is the flexibility coefficient, $\mathbf{T}$ is stress component, $\mathbf{E}$ is electric field, $\mathbf{d}$ is the piezoelectric constant, and $\boldsymbol{\varepsilon}^{T}$ is the free dielectric constant. In this paper, $\boldsymbol{s}^{E}$ is a complex expression including the loss factor $\eta_{s}$ in COMSOL. The mechanical loss factors $\eta_{s}$ of the titanium alloy, aluminum alloy, high-strength alloy steel and piezoelectric ceramics are assumed as $10^{-5}, 10^{-3}, 10^{-3}$, and $1.1 \times 10^{-2}$, respectively. The structural frame of the ultrasonic transducer is given a fixed constraint as the boundary condition.

\subsection{Acoustic field coupling in a gas-liquid medium}

In LFS, the vibration of the transducer head forms an acoustic field, and COMSOL presents a mathematical model to calculate the coupling relationship, that is, the Acoustic-Structure Boundary coupling to couple a Pressure Acoustics model with a Piezoelectric coupling model. The coupling includes the fluid load on the structure and the structural acceleration as experienced 
by the fluid. For the Acoustic-Structure Boundary, the boundary conditions in the fluid (the exterior boundary) satisfy the following equation:

$\left\{\begin{array}{l}-\mathbf{n} \cdot\left[-\frac{1}{\rho_{c}}\left(\nabla p_{t}\right)\right]=-\mathbf{n} \cdot \mathbf{u}_{t t} \\ \mathbf{F}_{A}=p_{t} \cdot \mathbf{n}\end{array}\right.$

where $\mathbf{u}_{t t}$ is the structural acceleration, $\mathbf{n}$ is the surface normal, $\rho_{c}$ is complex density of materials, $p_{t}$ is the total acoustic pressure and $\mathbf{F}_{A}$ is the load (force per unit area) experienced by the structure. In this case, $\mathbf{u}_{t t}$ and $\mathbf{F}_{A}$ are the results of the acoustic field and piezoelectric equations in Eq. (2). Then, $p_{t}$ can be calculated in terms of the initial and boundary conditions.

To obtain the total acoustic pressure $p_{t}$, the acoustic cavitation effect should be considered. Cavitation bubbles appear when the acoustic power of the ultrasonic transducer exceeds the cavitation threshold value. This gas-liquid medium changes the acoustic field distribution and acoustic pressure in the liquid. In this paper, a linear absorption coefficient $\alpha$ of sound propagation is used to represent the above acoustic field variation due to cavitation bubbles. Therefore, by introducing complex density $\rho_{c}$ and complex acoustic speed $c_{c}$ to explain the damping of the ultrasonic effect, the Helmholtz equation for the propagation of sound waves can be revised as:

$\nabla \frac{1}{\rho_{c}}\left(\nabla p_{t}\right)-\frac{k_{e q}^{2} p_{t}}{\rho_{c}}=0$

where:

$p_{t}=p+p_{b}, \quad k_{\alpha}=\frac{\omega}{c}-i \alpha, \quad \rho_{c}=\frac{\rho c^{2}}{c_{c}^{2}}, \quad c_{c}=\frac{\omega}{k_{\alpha}}, \quad k_{e q}^{2}=\left(\frac{\omega}{c_{c}}\right)^{2}=\left(\frac{\omega}{c}-i \alpha\right)^{2}$,

where $\omega=2 \pi f$ is the angular frequency, $p_{t}$ is the total acoustic pressure as a function of angular frequency $\omega$ at three-dimensional coordinates $(x, y, z), p_{b}$ is the undisturbed pressure in the liquid, $\alpha$ is the absorption coefficient, $\rho$ is the liquid density, and $c$ is the acoustic speed. The equivalent wavenumber $k_{e q}$ in the gas-liquid medium is equal to that in the acoustic field, namely $k_{\alpha}$, while considering sound attenuation.

\subsection{Bubbles dynamics}

In in-vitro LFS, many bubbles exist in the donor due to ultrasonic cavitation. According to the Cafisch equations theory proposed by Kerry W. Commander [8], the equivalent wavenumber $k_{e q}$ in the gas-liquid medium in an acoustic field should be:

$k_{e q}^{2}=\left(\frac{\omega}{c}\right)^{2}+4 \pi \omega^{2} \int_{0}^{\infty} \frac{R f(R)}{\omega_{0}^{2}-\omega^{2}+2 i b \omega} d R$

where $R$ is the equilibrium radium of cavitation bubbles and $f(R)$ is its certain radial distribution and volume fraction, which is assumed to be a Gaussian distribution. $\omega_{0}$ is the resonance frequency of the bubbles, $i$ is the imaginary unit, and $b$ is the damping factor. They are defined as [8]:

$f(R)= \begin{cases}C_{c o e} e^{-\left(R-R_{0}\right)^{2} / \sigma_{S D}^{2},} & R_{1}<R<R_{2} \\ 0, & \text { otherwise }\end{cases}$

$\omega_{0}^{2}=\frac{p_{b}}{\rho R^{2}}\left(R_{e} \Phi-\frac{2 \sigma_{1}}{R p_{b}}\right)$ 
$b=\frac{2 \mu_{1}}{\rho R^{2}}+\frac{p_{b}}{2 \rho R^{2}} \operatorname{Im} \Phi+\frac{\omega^{2} R}{2 c}$

where $C_{c o e}$ is a parameter chosen to match the gas volume void fraction $\beta, R_{0}$ is the radius for the Gaussian radii distribution with a maximum value, $\sigma_{S D}$ is a standard deviation of approximate $2 \times 10^{-3} \mathrm{~m}, R$ is in the range of bubble radii from $R_{1}=5 \times 10^{-6} \mathrm{~m}$ and $R_{2}=3 \times 10^{-3} \mathrm{~m}, \sigma_{l}$ is the surface tension of the liquid, $\mu_{l}$ is the viscosity of the liquid, and the undisturbed pressure in the bubble, equal to that in the liquid $p_{b}=p_{\infty}+2 \sigma_{l} / R$, while $p_{\infty}$ is the equilibrium pressure in the liquid. The complex dimensionless parameter $\Phi$ is defined as [8]:

$$
\Phi=\frac{3 \gamma}{1-3(\gamma-1) i \chi\left[\left(\frac{1}{\chi}\right)^{\frac{1}{2}} \operatorname{coth}\left(\frac{1}{\chi}\right)^{\frac{1}{2}}-1\right]},
$$

where $\gamma$ is the specific heat ratio of the gas inside the bubbles and $\chi=D / \omega R^{2}$, with $D$ as the thermal diffusivity of the gas. The relationship between the function $f(R)$ and gas volume void fraction $\beta$ is [8]:

$\beta(r, t)=\frac{4}{3} \pi \int_{0}^{\infty} R_{\text {instant }}^{3}(r, R, t) f(r, R) d R$,

where $R_{\text {instant }}(r, R, t)$ denotes the instantaneous bubble radius at time $t$ and position $r$ with an equilibrium radius $R$. In the experiment, the volume of the diffusion cell is small $(<20 \mathrm{~mL})$ enough to assume an average constant $\beta$ instead of $\beta(r, t)$ to calculate parameter $C_{c o e}$.

According to the same real part of wavenumber $k_{e q}$, in terms of Eq. (6), the absorption coefficient $\alpha$ can be represented as:

$\alpha=\sqrt{\operatorname{Re}\left[4 \pi \omega^{2} \int_{R_{1}}^{R_{2}} \frac{R f(R)}{\omega_{0}^{2}-\omega^{2}+2 i b \omega} d R\right]}$.

Therefore, Eqs. (6)-(10) can be substituted into Eq. (11) with the initial and boundary conditions, and the absorption coefficient $\alpha$ in LFS can be calculated. Then, the total acoustic pressure $p_{t}$ can be calculated in terms of Eqs. (2)-(4). The boundary conditions suggest that the liquid boundary contact with air is assumed to be a sound-soft boundary and a sound-stiff boundary with the glass wall.

\subsection{Finite element method}

In this paper, a piezoelectric-acoustic coupling model in COMSOL is used to calculate the acoustic distribution based on the above bubble dynamics results. The mesh of the model can be seen in Fig. 4. The ultrasonic transducer is calculated by piezoelectric equations of Eq. (2), and the acoustic fields are calculated by a Helmholtz equation of Eq. (4). The energy transfer functions are derived from the acoustic-structure boundaries on the acoustic exposure interface, as can be seen in Eq. (3). Due to the acoustic cavitation, there are plenty of bubbles in the donor, leading to a large absorption coefficient $\alpha$ of acoustic waves in the drug liquid. The absorption coefficient $\alpha$ can be calculated by Eqs. (5)-(11) in terms of bubble dynamics, and then it will be substituted into the Eq. (4) which is used to calculate the FEM model of acoustic pressure.

In our experiments, the skin thickness was $d=5.62 \times 10^{-4} \mathrm{~m}$ and the absorption coefficient $\alpha_{2}$ of skin was assumed to be $4 \mathrm{~Np} / \mathrm{m}$ at $21 \mathrm{kHz}$. The initial boundary condition is the input voltage on piezoelectric ceramics, which is $V_{0 p}=40 \mathrm{~V}$. The glass walls of the in vitro LFS are all set as 
hard acoustic boundaries, and the air-liquid interface is set as a soft acoustic boundary. Therefore, with the above calculation methods and boundary conditions, the acoustic fields can be calculated based on FEM in terms of the absorption coefficient $\alpha$ derived from bubbly dynamics.

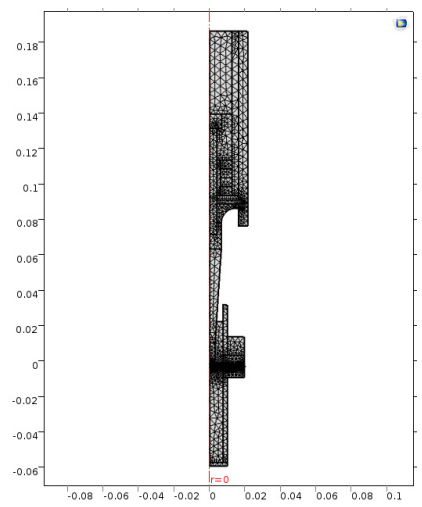

Fig. 4. FEM mesh

\section{Results and discussion}

First, a piezoelectric coupling calculation of the ultrasonic transducer was executed using COMSOL. The simulated displacement amplitude in the air of the transducer head was calculated according to Eq. (2), and it was also measured by a PSV-500 scanning vibrometer at $5.5 \mathrm{~W}$ and an electric input power of $21 \mathrm{kHz}$, as shown in Fig. 5. For the simulation, the boundary condition and material parameters are presented in Section 3.4 and the material database is presented in COMSOL; the initial boundary condition is $V_{0 p}=29 \mathrm{~V}$ (that is, $5.5 \mathrm{~W}$ input power in the air). Fig. 5 illustrates that the measured displacement amplitudes of the transducer head are relatively equal to the simulation amplitudes and that the entire surface vibration results are approximately $16 \mu \mathrm{m}$ in the air. Therefore, this piezoelectric coupling calculation method can be effectively applied in the next step on the acoustic field simulation.

After the piezoelectric coupling process is completed, the acoustic field in in vitro LFS can be calculated according to Section 3.2 and 3.3. Then, according to Eqs. (5)-(10) and the Cafisch theory [8], the complex dimensionless parameter $\Phi$ can be calculated as seen in Fig. 6(a), and the damping factor $b$ is shown in Fig. 6(b). In the experiments, there were sodium lauryl sulfate (a surfactant used to synergistically enhance the effects of sonophoresis) in the donor but almost none in the receptor, which can dramatically enhance the gas volume void fraction. So, given the gas volume void fraction $\beta=10^{-3}$ as the approximate average value in the donor at $21 \mathrm{kHz}$ and $\beta=10^{-5}$ in the receptor, approximate values are chosen from document [8] to represent the large number of bubbles in the donor and smaller number of bubbles in the receptor. Therefore, the absorption coefficient $\alpha$ can be numerically calculated to be $29.4 \mathrm{~Np} / \mathrm{m}$ and $2.9 \mathrm{~Np} / \mathrm{m}$ in the donor and the receptor, respectively.

The acoustic pressure $p_{t}$ in a Franz diffusion cell can then be calculated based on Eq. (4) at $V_{0 p}$ of $40 \mathrm{~V}(5.5 \mathrm{~W}$ electric power input in water) and $21 \mathrm{kHz}$, and its results are presented in Fig. 7. Fig. 7(a) shows that in the entire acoustic pressure distribution (simulation) of LFS, the maximum value is $2.01 \times 10^{5} \mathrm{~Pa}$, which appears under the transducer head; the acoustic pressure declined sharply as it is away from the head. Fig. 7(b) and (c) describe the acoustic pressure distribution (simulation) on both the stratum corneum and dermis of the skin, respectively, and the maximum value of which is $5.1 \times 10^{4} \mathrm{~Pa}$, which appears on the stratum corneum under the center of the transducer head, while the maximum value on the dermis surface in the receptor is $3.8 \times 10^{4} \mathrm{~Pa}$. Fig. 7(d) illustrates the acoustic pressure distribution (simulation) at the position of liquid draw-off in the receptor, and the pressure values are all approximately $2.0 \times 10^{3} \mathrm{~Pa}$. 


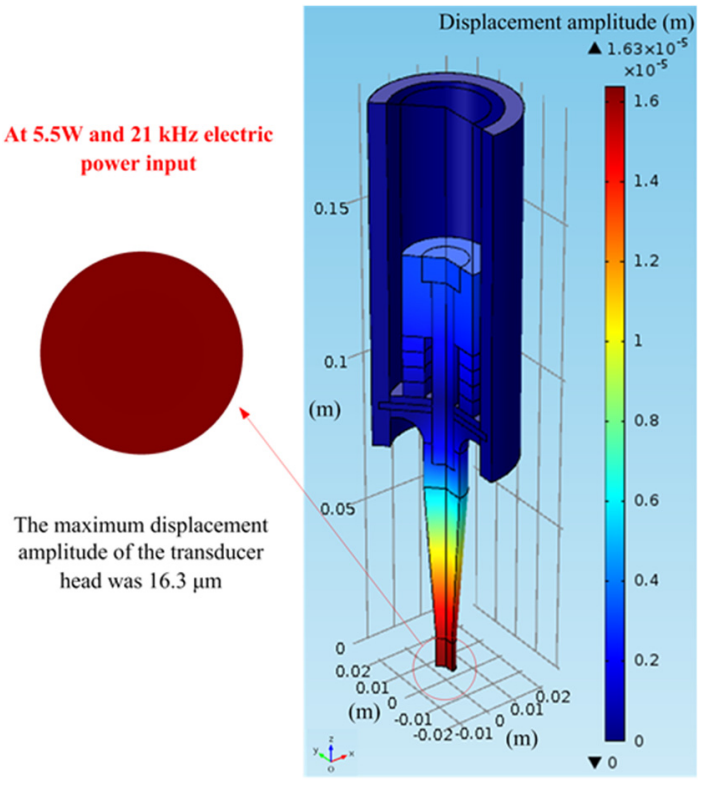

a)

Fig. 5. Vibration displacement amplitude on the top of the transducer head at $5.5 \mathrm{~W}$

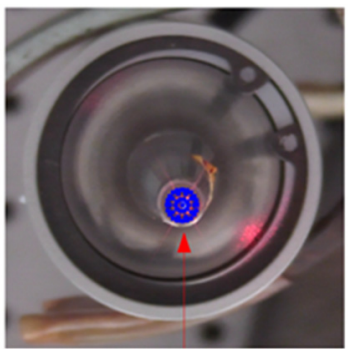

Test points in the top of transducer head

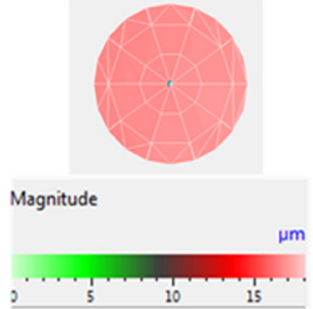

Displacement distribution and the maximum displacement amplitude of the transducer head was $16.7 \mu \mathrm{m}$

b) and $21 \mathrm{kHz}$ in air: a) simulated results; b) experimental results

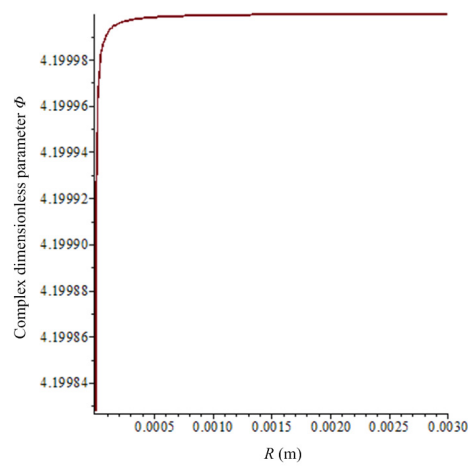

a)

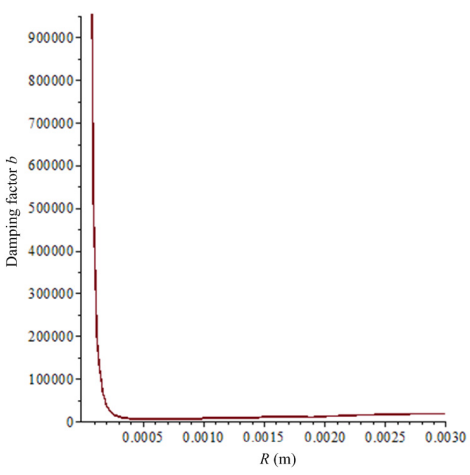

b)

Fig. 6. Calculation parameters relationship with $R$ (the equilibrium radium of cavitation bubbles):

a) complex dimensionless parameter $\Phi$; b) damping factor $b$

Further, in terms of Eq. (1), the experimental acoustic pressure under the transducer head is $9.1( \pm 0.2) \times 10^{4} \mathrm{~Pa}$ when the measured response voltage of the PVDF strip is $4.2 \pm 0.9 \mathrm{~V}$; the acoustic pressure at the liquid draw-off position is $2.1( \pm 0.6) \times 10^{3} \mathrm{~Pa}$ when the PVDF response voltage is $0.097 \pm 0.026 \mathrm{~V}$. As shown in Fig. 7(b) and (d), the simulation acoustic pressure value on the parallel plane at the liquid draw-off position is approximately equal to the experiment value, but the one on the skin is smaller than the experiment value, this is because of the thickness influence of the PVDF strip. The actual measurement position is about $2 \mathrm{~mm}$ above the skin.

In LFS, the distance between the skin and transducer head, investigated in Fig. 8, is an important factor in permeation efficiency. In the measurement, the skin, PVDF strip, and Franz cell were fixed together and the height of the ultrasonic transducer was adjusted to change the distance between the skin and its head. The PVDF piezoelectric strip in the donor was used to measure the acoustic pressure, following the steps described in Section 2. The figure describes the maximum acoustic pressure with different heights at $V_{0 p}=40 \mathrm{~V}$. In Fig. 8, both the experimental 
and simulation results show a quick reduction in the acoustic pressure with an increase in distance, and simulation results are approximately close to the experiment values. Nevertheless, there is some deviation between experimental and simulation values, this is because the acoustic field was slightly changed by the PVDF strip as it was used as measure tool. The above results demonstrate that the height of ultrasonic head is very sensitive to the acoustic pressure on the skin in LFS, so the head should be put closer to the skin as much as possible.

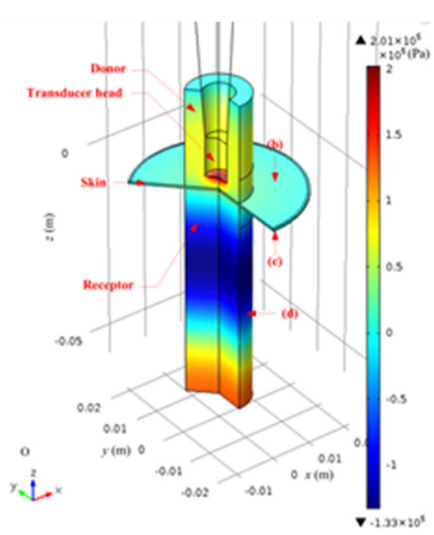

a)

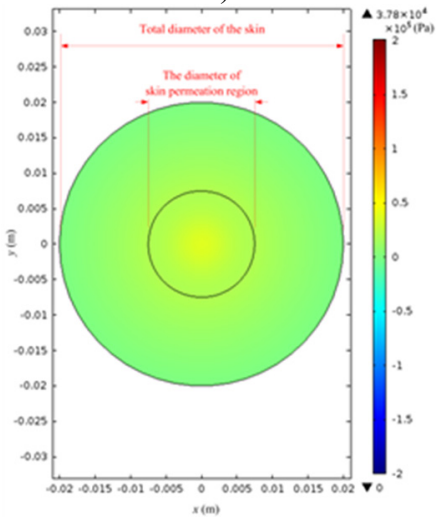

c)

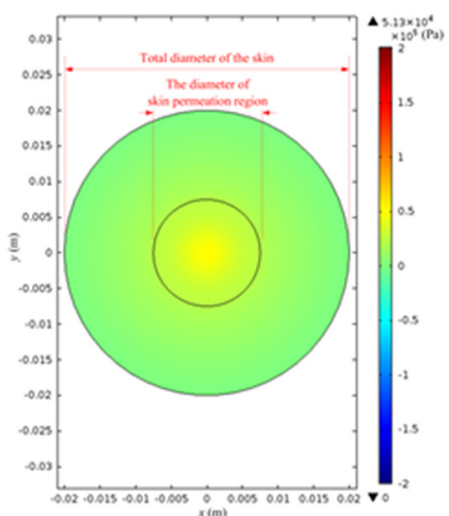

b)

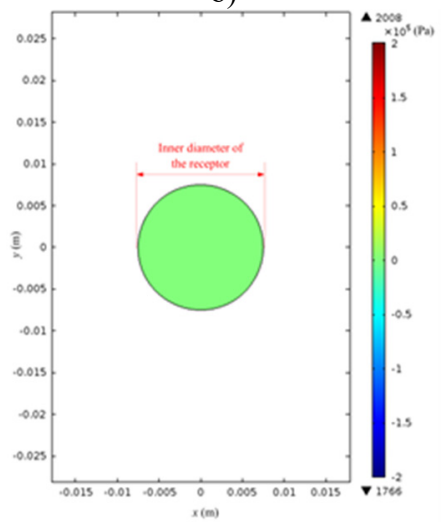

d)

Fig. 7. Simulated acoustic pressure distribution at $V_{0 p}=40 \mathrm{~V}$ : a) acoustic pressure distribution in the Franz diffusion cell; b) acoustic pressure distribution at the upper surface of the skin in the donor; c) acoustic pressure distribution at the lower surface of the skin in the receptor;

d) acoustic pressure distribution on the parallel plane at the liquid draw-off position

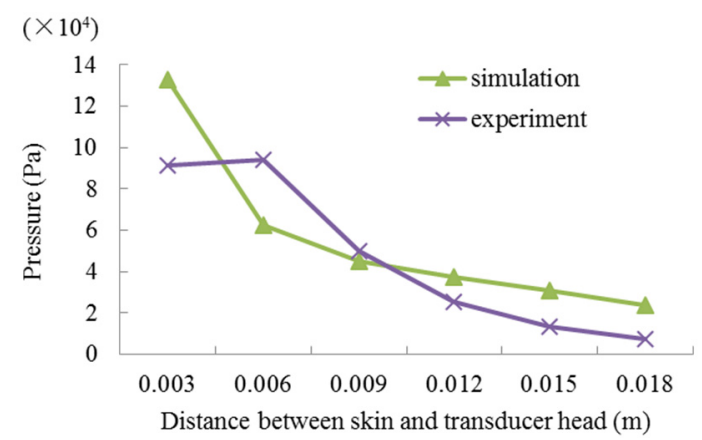

Fig. 8. Maximum acoustic pressure at $2 \mathrm{~mm}$ height upon the skin with different distance between the skin and transducer head at $V_{0 p}=40 \mathrm{~V}$ 


\section{Conclusions}

For obtaining the acoustic pressure distribution in LFS, an acoustic-piezoelectric coupling model is established based on COMSOL multiphysics. Aiming at acoustic cavitations effect, the absorption coefficients of liquids are calculated in terms of bubble dynamics. Experimental and calculated results show that calculated acoustic pressure is appropriately close to the experimental values; the maximum acoustic pressure appears under the transducer's head, and it quickly decays far away from the head; the transducer should be put closer to the skin for larger pressure. Therefore, this model can be utilized in the analyses of energy transformation in LFS.

\section{Acknowledgements}

This work is supported by the following funding organizations in China: The National Natural Science Foundation of China (Grant No. 51405224), the Natural Science Foundation of Jiangsu Province (Grant No. BK20140818), the Fundamental Research Funds for the Central Universities (Grant No. NJ20160003).

\section{References}

[1] Park D., Park H., Seo J., et al. Sonophoresis in transdermal drug deliveries. Ultrasonics, Vol. 54, Issue 1, 2014, p. 56-65.

[2] Azagury A., Khoury L., Enden G., et al. Ultrasound mediated transdermal drug delivery. Advanced Drug Delivery Reviews, Vol. 72, 2014, p. 127-143.

[3] Seto J. E., Polat B. E., Lopez R. F. V., et al. Effects of ultrasound and sodium lauryl sulfate on the transdermal delivery of hydrophilic permeants: comparative in vitro studies with full-thickness and split-thickness pig and human skin. Journal of Controlled Release, Vol. 145, Issue 1, 2010, p. 26-32.

[4] Polat B. E., Blankschtein D., Langer R. Low-frequency sonophoresis: application to the transdermal delivery of macromolecules and hydrophilic drugs. Expert Opinion on Drug Delivery, Vol. 7, Issue 12, 2010, p. 1415-1432.

[5] Mitragotri S., Blankschrein D., Langer R. Ultrasound-mediated transdermal protein delivery. Science, Vol. 269, 5225, p. 1995-850.

[6] Aldwaikat M., Alarjah M. Investigating the sonophoresis effect on the permeation of diclofenac sodium using 3D skin equivalent. Ultrasonics Sonochemistry, Vol. 22, 2015, p. 580-587.

[7] Wolloch L., Kost J. The importance of microjet vs shock wave formation in sonophoresis. Journal of Controlled Release, Vol. 148, Issue 2, 2010, p. 204-211.

[8] Commander K. W., Prosperetti A. Linear pressure waves in bubbly liquids: Comparison between theory and experiments. Journal of The Acoustical Society of America, Vol. 2, Issue 85, 1989, p. $732-746$.

[9] Zhao C. Ultrasonic Motors: Technologies and Applications. First Ed. Science Press Beijing and Springer, Beijing, 2011, p. 28-31.

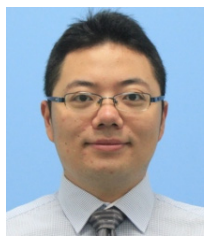

Hanmin Peng received Ph.D. degree in Electromechanical Engineering Institute from Nanjing University of Aeronautics and Astronautics, Nanjing, China, in 2011. Now he works at this university. His current research interests include ultrasonic vibration, piezoelectric sensors and actuators.

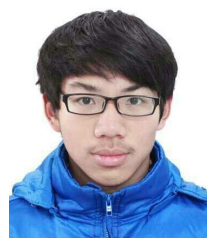

Penghui Lu is an undergraduate student, Major in aircraft design in Nanjing University of Aeronautics and Astronautics. His current research interests include vibration, piezoelectric actuators and structural design. 


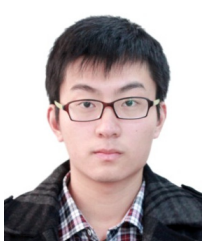

Pancheng Zhu received B.Eng. degree in College of Aerospace Engineering from Nanjing University of Aeronautics and Astronautics, Nanjing, China, in 2016. Now he is studying for a Master's degree at this university. His current research interests include ultrasonic vibration, piezoelectric actuators and ultrasonic motors.

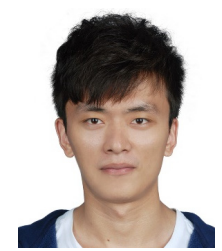

Boping $\mathbf{Y u}$ is an undergraduate student, Major in aircraft design in Nanjing University of Aeronautics and Astronautics. His current research interests include vibration and piezoelectric actuators. 